\title{
A generalization of the modified Simpson's rule and error bounds
}

\author{
Nenad Ujević
}

(Received 4 October 2004)

\begin{abstract}
A generalization of the modified Simpson's rule is derived. Various error bounds for this generalization are established. An application to Dawson integral is given.
\end{abstract}

\section{Contents}

1 Introduction

2 Main results

3 A numerical example

*Department of Mathematics, University of Split, Split, Croatia. mailto:ujevic@pmfst.hr

See http://anziamj . austms .org.au/V47E/Ujevic for this article, C) Austral. Mathematical Soc. 2005. Published November 9, 2005. ISSN 1446-8735
E2

E3

E11 


\section{Introduction}

In recent years a number of authors have considered generalizations of some known and some new quadrature rules. For example, generalizations of the trapezoid, mid-point and Simpson's quadrature rules are considered in [1, $2,3,4,5]$. As an illustration we give a generalization of the mid-point quadrature rule [2],

$$
\begin{aligned}
\int_{a}^{b} f(t) d t= & \sum_{k=0}^{n-1}\left[1+(-1)^{k}\right] \frac{(b-a)^{k+1}}{2^{k+1}(k+1) !} f^{(k)}\left(\frac{a+b}{2}\right) \\
& +(-1)^{n} \int_{a}^{b} K_{n}(t) f^{(n)}(t) d t
\end{aligned}
$$

where

$$
K_{n}(t)= \begin{cases}\frac{(t-a)^{n}}{n !}, & t \in\left[a, \frac{a+b}{2}\right], \\ \frac{(t-b)^{n}}{n !}, & t \in\left(\frac{a+b}{2}, b\right] .\end{cases}
$$

For $n=1$ we get the mid-point rule,

$$
\int_{a}^{b} f(t) d t=(b-a) f\left(\frac{a+b}{2}\right)-\int_{a}^{b} K_{1}(t) f^{\prime}(t) d t .
$$

In this paper we consider a generalization of the modified Simpson's rule:

$$
\begin{aligned}
\int_{a}^{b} f(x) d x= & \frac{b-a}{30}\left[7 f(a)+16 f\left(\frac{a+b}{2}\right)+7 f(b)\right] \\
& -\frac{(b-a)^{2}}{60}\left[f^{\prime}(b)-f^{\prime}(a)\right]+\bar{R}(f),
\end{aligned}
$$


where the error term is, to a leading order estimate,

$$
\bar{R}(f) \approx \frac{(b-a)^{6}}{302400}\left[f^{v}(b)-f^{v}(a)\right] .
$$

The above rule is derived in [6]. It is shown that this rule has much better approximation properties than the well-known classical Simpson's rule. In [6] we also find various error inequalities for this rule.

Here we give a generalization of of the modified Simpson's rule and we also give various error bounds for the generalization . These error bounds are generalizations of the error bounds obtained in [6].

Finally, we give a numerical example: we derive a summation formula for the Dawson integral $E(x)=\int_{0}^{x} \exp \left(t^{2}\right) d t$.

\section{Main results}

Theorem 1 Let $f:[a, b] \rightarrow R$ be a function such that $f^{(n-1)}$ is absolutely continuous. Then

$$
\begin{gathered}
\int_{a}^{b} f(x) d x=\frac{7 f(a)+16 f\left(\frac{a+b}{2}\right)+7 f(b)}{30}(b-a)-\frac{f^{\prime}(b)-f^{\prime}(a)}{60}(b-a)^{2} \\
+\frac{1}{15} \sum_{i=3}^{m} \frac{(i-1)(i-2)(b-a)^{2 i+1}}{2^{2 i-2}(2 i+1) !} f^{(2 i)}\left(\frac{a+b}{2}\right)+R(f),
\end{gathered}
$$

where $m=\left[\frac{n-1}{2}\right]$, the integer part of $(n-1) / 2$,

$$
R(f)=(-1)^{n} \int_{a}^{b} S_{n}(t) f^{(n)}(t) d t
$$


and

$$
S_{n}(t)= \begin{cases}\frac{(t-a)^{n-2}}{n !}\left[(t-a)^{2}-\frac{7 n(b-a)(t-a)}{30}+\frac{n(n-1)(b-a)^{2}}{60}\right], & t \in\left[a, \frac{a+b}{2}\right], \\ \frac{(t-b)^{n-2}}{n !}\left[(t-b)^{2}+\frac{7 n(b-a)(t-b)}{30}+\frac{n(n-1)(b-a)^{2}}{60}\right], & t \in\left(\frac{a+b}{2}, b\right] .\end{cases}
$$

Proof: We prove (1) by induction. First we note that

$$
\begin{aligned}
& S_{2}(t)= \begin{cases}\frac{1}{2}(t-a)^{2}-\frac{7(b-a)(t-a)}{15}+\frac{(b-a)^{2}}{30}, & t \in\left[a, \frac{a+b}{2}\right], \\
\frac{1}{2}(t-b)^{2}+\frac{7(b-a)(t-b)}{15}+\frac{(b-a)^{2}}{30}, & t \in\left(\frac{a+b}{2}, b\right] ;\end{cases} \\
& S_{3}(t)= \begin{cases}\frac{(t-a)}{3 !}\left[(t-a)^{2}-\frac{7(b-a)(t-a)}{10}+\frac{(b-a)^{2}}{10}\right], & t \in\left[a, \frac{a+b}{2}\right], \\
\frac{(t-b)}{3 !}\left[(t-b)^{2}+\frac{7(b-a)(t-b)}{10}+\frac{(b-a)^{2}}{10}\right], & t \in\left(\frac{a+b}{2}, b\right] ;\end{cases} \\
& S_{4}(t)= \begin{cases}\frac{(t-a)^{2}}{4 !}\left[(t-a)^{2}-\frac{14(b-a)(t-a)}{15}+\frac{(b-a)^{2}}{5}\right], & t \in\left[a, \frac{a+b}{2}\right], \\
\frac{(t-b)^{2}}{4 !}\left[(t-b)^{2}+\frac{14(b-a)(t-b)}{15}+\frac{(b-a)^{2}}{5}\right], & t \in\left(\frac{a+b}{2}, b\right] ;\end{cases} \\
& S_{5}(t)= \begin{cases}\frac{(t-a)^{3}}{5 !}\left[(t-a)^{2}-\frac{7(b-a)(t-a)}{6}+\frac{(b-a)^{2}}{3}\right], & t \in\left[a, \frac{a+b}{2}\right], \\
\frac{(t-b)^{3}}{5 !}\left[(t-b)^{2}+\frac{7(b-a)(t-b)}{6}+\frac{(b-a)^{2}}{3}\right], & t \in\left(\frac{a+b}{2}, b\right] ;\end{cases} \\
& S_{6}(t)= \begin{cases}\frac{(t-a)^{4}}{6 !}\left[(t-a)^{2}-\frac{7(b-a)(t-a)}{5}+\frac{(b-a)^{2}}{2}\right], & t \in\left[a, \frac{a+b}{2}\right], \\
\frac{(t-b)^{4}}{6 !}\left[(t-b)^{2}+\frac{7(b-a)(t-b)}{5}+\frac{(b-a)^{2}}{2}\right], & t \in\left(\frac{a+b}{2}, b\right] ;\end{cases}
\end{aligned}
$$

are Peano kernels for the modified Simpson's quadrature rule, that is, we have

$$
\begin{aligned}
\int_{a}^{b} S_{6}(t) f^{(6)}(t) d t & =-\int_{a}^{b} S_{5}(t) f^{(5)}(t) d t \\
& =\int_{a}^{b} S_{4}(t) f^{(4)}(t) d t \\
& =-\int_{a}^{b} S_{3}(t) f^{\prime \prime \prime}(t) d t
\end{aligned}
$$




$$
\begin{aligned}
= & \int_{a}^{b} S_{2}(t) f^{\prime \prime}(t) d t \\
= & -\frac{7 f(a)+16 f\left(\frac{a+b}{2}\right)+7 f(b)}{30}(b-a) \\
& +\frac{f^{\prime}(b)-f^{\prime}(a)}{60}(b-a)^{2}+\int_{a}^{b} f(t) d t .
\end{aligned}
$$

We easily show that (1) holds for $n=7$. Now suppose that (1) holds for an arbitrary $n$. We have to prove that (1) holds for $n \rightarrow n+1$. To simplify the proof we introduce

$$
\begin{aligned}
& P_{n}(t)=\frac{(t-a)^{n-2}}{n !}\left[(t-a)^{2}-\frac{7 n(b-a)(t-a)}{30}+\frac{n(n-1)(b-a)^{2}}{60}\right], \\
& Q_{n}(t)=\frac{(t-b)^{n-2}}{n !}\left[(t-b)^{2}+\frac{7 n(b-a)(t-b)}{30}+\frac{n(n-1)(b-a)^{2}}{60}\right] .
\end{aligned}
$$

We see that $P_{n}$ and $Q_{n}$ form Appell sequences of polynomials, that is

$$
P_{n}^{\prime}(t)=P_{n-1}(t), \quad Q_{n}^{\prime}(t)=Q_{n-1}(t) .
$$

Then we have

$$
\begin{aligned}
& (-1)^{n+1} \int_{a}^{b} S_{n+1}(t) f^{(n+1)}(t) d t \\
& =(-1)^{n+1} \int_{a}^{\frac{a+b}{2}} P_{n+1}(t) f^{(n+1)}(t) d t+(-1)^{n+1} \int_{\frac{a+b}{2}}^{b} Q_{n+1}(t) f^{(n+1)}(t) d t \\
& =(-1)^{n+1}\left[P_{n+1}\left(\frac{a+b}{2}\right) f^{(n)}\left(\frac{a+b}{2}\right)-P_{n+1}(a) f^{(n)}(a)\right] \\
& \quad+(-1)^{n} \int_{a}^{\frac{a+b}{2}} P_{n}(t) f^{(n)}(t) d t \\
& \quad+(-1)^{n+1}\left[Q_{n+1}(b) f^{(n)}(b)-Q_{n+1}\left(\frac{a+b}{2}\right) f^{(n)}\left(\frac{a+b}{2}\right)\right]
\end{aligned}
$$




$$
\begin{aligned}
& +(-1)^{n} \int_{\frac{a+b}{2}}^{b} Q_{n}(t) f^{(n)}(t) d t \\
= & (-1)^{n} \int_{a}^{b} S_{n}(t) f^{(n)}(t) d t \\
& +(-1)^{n+1}\left[P_{n+1}\left(\frac{a+b}{2}\right)-Q_{n+1}\left(\frac{a+b}{2}\right)\right] f^{(n)}\left(\frac{a+b}{2}\right) \\
= & -\frac{7 f(a)+16 f\left(\frac{a+b}{2}\right)+7 f(b)}{30}(b-a)+\frac{f^{\prime}(b)-f^{\prime}(a)}{60}(b-a)^{2} \\
& +\int_{a}^{b} f(t) d t-\frac{1}{15} \sum_{i=3}^{m} \frac{(i-1)(i-2)(b-a)^{2 i+1}}{2^{2 i-2}(2 i+1) !} f^{(2 i)}\left(\frac{a+b}{2}\right) \\
& +(-1)^{n+1}\left[P_{n+1}\left(\frac{a+b}{2}\right)-Q_{n+1}\left(\frac{a+b}{2}\right)\right] f^{(n)}\left(\frac{a+b}{2}\right) \\
= & -\frac{7 f(a)+16 f\left(\frac{a+b}{2}\right)+7 f(b)}{30}(b-a)+\frac{f^{\prime}(b)-f^{\prime}(a)}{60}(b-a)^{2} \\
& +\int_{a}^{b} f(t) d t-\frac{1}{15} \sum_{i=3}^{m_{1}} \frac{(i-1)(i-2)(b-a)^{2 i+1}}{2^{2 i-2}(2 i+1) !} f^{(2 i)}\left(\frac{a+b}{2}\right),
\end{aligned}
$$

where $m_{1}=\left[\frac{n}{2}\right]$, since

$$
\begin{aligned}
& (-1)^{n+1}\left[P_{n+1}\left(\frac{a+b}{2}\right)-Q_{n+1}\left(\frac{a+b}{2}\right)\right] f^{(n)}\left(\frac{a+b}{2}\right) \\
& =(-1)^{n+1} \frac{(b-a)^{n+1}}{2^{n+1}(n+1) !}\left[1-(-1)^{n+1}\right] \frac{(n-2)(n-4)}{15} f^{(n)}\left(\frac{a+b}{2}\right) .
\end{aligned}
$$

This completes the proof.

Lemma 2 The Peano kernels $S_{n}(t), n>6$, satisfy:

$$
\int_{a}^{b} S_{n}(t) d t=0, \quad n \text { is odd }
$$




$$
\begin{aligned}
& \int_{a}^{b}\left|S_{n}(t)\right| d t=\frac{(n-2)(n-4)}{15} \frac{(b-a)^{n+1}}{2^{n}(n+1) !}, \\
& \max _{t \in[a, b]}\left|S_{n}(t)\right|=\frac{(n-3)(n-5)}{15} \frac{(b-a)^{n}}{2^{n} n !} .
\end{aligned}
$$

Proof: A simple calculation gives

$$
\int_{a}^{b} S_{n}(t) d t=\frac{(b-a)^{n+1}}{2^{n+1}(n+1) !} \frac{(n-2)(n-4)}{15}\left[1-(-1)^{n+1}\right] .
$$

From the above relation we see that (6) holds, since $1-(-1)^{n+1}=0$ if $n$ is odd.

We now consider some properties of the Appell sequences of polynomials $P_{n}(t)$ and $Q_{n}(t)$, given by (4) and (5), respectively. We have that $(t-a)^{n-2} \geq$ 0 , for each $n>6$ and $t \in\left[a, \frac{a+b}{2}\right]$. We also have

$$
(t-a)^{2}-\frac{7 n(b-a)(t-a)}{30}+\frac{n(n-1)(b-a)^{2}}{60} \geq 0, \quad t \in\left[a, \frac{a+b}{2}\right], \quad n>6,
$$

such that $P_{n}(t) \geq 0$ for $n>6$ and $t \in\left[a, \frac{a+b}{2}\right]$. Since $P_{n}^{\prime}(t)=P_{n-1}(t)$ we conclude that $P_{n}(t)$ are increasing functions.

We have that $(t-b)^{n-2} \leq 0$ if $n>6, n$ is odd and $(t-b)^{n-2} \geq 0$ if $n>6$, $n$ is even. Since

$$
(t-b)^{2}+\frac{7 n(b-a)(t-b)}{30}+\frac{n(n-1)(b-a)^{2}}{60} \geq 0, \quad t \in\left[\frac{a+b}{2}, b\right], \quad n>6,
$$

we have that $Q_{n}(t) \leq 0$ if $n$ is odd and $Q_{n}(t) \geq 0$ if $n$ is even. As we know $Q_{n}^{\prime}(t)=Q_{n-1}(t)$ such that $Q_{n}(t)$ are decreasing functions if $n$ is even and $Q_{n}(t)$ are increasing functions if $n$ is odd. We use these properties to prove (7) and (8). 
We have

$$
\begin{aligned}
\int_{a}^{b}\left|S_{n}(t)\right| d t & =\int_{a}^{\frac{a+b}{2}}\left|P_{n}(t)\right| d t+\int_{\frac{a+b}{2}}^{b}\left|Q_{n}(t)\right| d t \\
& =\left|\int_{a}^{\frac{a+b}{2}} P_{n}(t) d t\right|+\left|\int_{\frac{a+b}{2}}^{b} Q_{n}(t) d t\right| \\
& =\frac{(n-2)(n-4)}{15} \frac{(b-a)^{n+1}}{2^{n}(n+1) !} .
\end{aligned}
$$

Finally, we have

$$
\begin{aligned}
\max _{t \in[a, b]}\left|S_{n}(t)\right| & =\max \left\{\max _{t \in\left[a, \frac{a+b}{2}\right]}\left|P_{n}(t)\right|, \max _{t \in\left[\frac{a+b}{2}, b\right]}\left|Q_{n}(t)\right|\right\} \\
& =\max \left\{\left|P_{n}\left(\frac{a+b}{2}\right)\right|,\left|Q_{n}\left(\frac{a+b}{2}\right)\right|\right\} \\
& =\frac{(n-3)(n-5)}{15} \frac{(b-a)^{n}}{2^{n} n !} .
\end{aligned}
$$

We introduce the notation

$$
\begin{aligned}
I= & \int_{a}^{b} f(t) d t \\
F= & \frac{7 f(a)+16 f\left(\frac{a+b}{2}\right)+7 f(b)}{30}(b-a)-\frac{f^{\prime}(b)-f^{\prime}(a)}{60}(b-a)^{2} \\
& +\frac{1}{15} \sum_{i=3}^{m} \frac{(i-1)(i-2)(b-a)^{2 i+1}}{2^{2 i-2}(2 i+1) !} f^{(2 i)}\left(\frac{a+b}{2}\right) .
\end{aligned}
$$

Theorem 3 Let $f:[a, b] \rightarrow R$ be a function such that $f^{(n-1)}, n>6$, is absolutely continuous and there exist real numbers $\gamma_{n}, \Gamma_{n}$ such that $\gamma_{n} \leq$ 
$f^{(n)}(t) \leq \Gamma_{n}, t \in[a, b]$. Then

$$
|I-F| \leq \frac{\Gamma_{n}-\gamma_{n}}{30(n+1) !} \frac{(n-2)(n-4)}{2^{n}}(b-a)^{n+1} \quad \text { if } n \text { is odd, }
$$

and

$$
|I-F| \leq \frac{1}{15} \frac{(b-a)^{n+1}(n-2)(n-4)}{2^{n}(n+1) !}\left\|f^{(n)}\right\|_{\infty} \quad \text { if } n \text { is even. }
$$

Proof: Let $n$ be odd. From (2) and (6) we get

$$
R(f)=(-1)^{n} \int_{a}^{b} S_{n}(t) f^{(n)}(t) d t=(-1)^{n} \int_{a}^{b} S_{n}(t)\left[f^{(n)}(t)-\frac{\gamma_{n}+\Gamma_{n}}{2}\right] d t
$$

such that we have

$$
|R(f)|=|I-F| \leq \max _{t \in[a, b]}\left|f^{(n)}(t)-\frac{\gamma_{n}+\Gamma_{n}}{2}\right| \int_{a}^{b}\left|S_{n}(t)\right| d t .
$$

We also have

$$
\max _{t \in[a, b]}\left|f^{(n)}(t)-\frac{\gamma_{n}+\Gamma_{n}}{2}\right| \leq \frac{\Gamma_{n}-\gamma_{n}}{2} .
$$

From (11), (12) and (7) we get

$$
|I-F| \leq \frac{\Gamma_{n}-\gamma_{n}}{30(n+1) !} \frac{(n-2)(n-4)}{2^{n}}(b-a)^{n+1} .
$$

Let $n$ be even. Then we have

$$
\begin{aligned}
|R(f)| & =|I-F| \\
\leq & \int_{a}^{b}\left|S_{n}(t)\right| d t\left\|f^{(n)}\right\|_{\infty} \\
& =\frac{1}{15} \frac{(b-a)^{n+1}(n-2)(n-4)}{2^{n}(n+1) !}\left\|f^{(n)}\right\|_{\infty} .
\end{aligned}
$$


Theorem 4 Let $f:[a, b] \rightarrow R$ be a function such that $f^{(n-1)}, n>6$, is absolutely continuous and let $n$ be odd. If there exists a real number $\gamma_{n}$ such that $\gamma_{n} \leq f^{(n)}(t), t \in[a, b]$ then

$$
|I-F| \leq \frac{1}{15}\left(T_{n}-\gamma_{n}\right) \frac{(n-3)(n-5)(b-a)^{n+1}}{2^{n} n !},
$$

where

$$
T_{n}=\frac{f^{(n-1)}(b)-f^{(n-1)}(a)}{b-a} .
$$

If there exists a real number $\Gamma_{n}$ such that $f^{(n)}(t) \leq \Gamma_{n}, t \in[a, b]$ then

$$
|I-F| \leq \frac{1}{15}\left(\Gamma_{n}-T_{n}\right) \frac{(n-3)(n-5)(b-a)^{n+1}}{2^{n} n !} .
$$

Proof: We have

$$
|R(f)|=|I-F|=\left|\int_{a}^{b}\left(f^{(n)}(t)-\gamma_{n}\right) S_{n}(t) d t\right|,
$$

since (6) holds. Then we have

$$
\begin{aligned}
& \left|\int_{a}^{b}\left(f^{(n)}(t)-\gamma_{n}\right) S_{n}(t) d t\right| \\
& \leq \max _{t \in[a, b]}\left|S_{n}(t)\right| \int_{a}^{b}\left(f^{(n)}(t)-\gamma_{n}\right) d t \\
& =\frac{1}{15} \frac{(n-3)(n-5)(b-a)^{n}}{2^{n} n !}\left[f^{(n-1)}(b)-f^{(n-1)}(a)-\gamma_{n}(b-a)\right] \\
& =\frac{1}{15}\left(T_{n}-\gamma_{n}\right) \frac{(n-3)(n-5)(b-a)^{n+1}}{2^{n} n !} .
\end{aligned}
$$

In a similar way we can prove that (14) holds.

Remark 5 Note that we can apply the estimations (9) and (10) only if $f^{(n)}$ is bounded. On the other hand, we can apply the estimation (13) if $f^{(n)}$ is unbounded above and we can apply the estimation (14) if $f^{(n)}$ is unbounded below. 


\section{A numerical example}

Here we consider the integral (Dawson integral) $E(x)=\int_{0}^{x} \exp \left(t^{2}\right) d t$ and apply the summation formula (1) to this integral. We get the summation formula $E(x)=F(x)+R(x)$, where

$$
\begin{aligned}
F(x)= & \frac{x}{30}\left[7+16 \exp \left(\frac{x^{2}}{4}\right)+7 \exp \left(x^{2}\right)\right]-\frac{x^{3} \exp \left(x^{2}\right)}{30} \\
& +\frac{1}{15} \sum_{i=3}^{m} \frac{(i-1)(i-2) x^{2 i+1}}{2^{2 i-2}(2 i+1) !} f^{(2 i)}\left(\frac{x}{2}\right)
\end{aligned}
$$

and $f(t)=\exp \left(t^{2}\right)$. We calculate the derivatives $f^{(j)}(t)$ :

$$
f^{(j)}(t)=P_{j}(t) \exp \left(t^{2}\right), \quad P_{j}(t)=\sum_{i=0}^{j} a_{j i} t^{i},
$$

where $P_{j}(t)$ is an odd function if $j$ is odd and $P_{j}(t)$ is an even function if $j$ is even. The coefficients $a_{j i}$ are

$$
\begin{aligned}
& a_{j i}=0, \quad j=1,2, \ldots, \quad i=0,1,2, \ldots, j, \\
& a_{11}=2, \quad a_{20}=2, \quad a_{2,2}=4, \\
& a_{j 1}=a_{j-1,2}, \quad a_{j i}=2 a_{j-1, i-1}+(j+1) a_{j-1, i+1}, \quad a_{j j}=2^{j} .
\end{aligned}
$$

We now compare the summation formula (15) with the known compound formula (for the modified Simpson's rule),

$$
\begin{aligned}
\int_{0}^{x} f(t) d t= & \frac{7 h}{30}[1+f(x)]+\frac{7 h}{15} \sum_{i=1}^{n-1} f\left(x_{i}\right)+\frac{8 h}{15} \sum_{i=0}^{n-1} f\left(\frac{x_{i}+x_{i+1}}{2}\right) \\
& -\frac{x \exp \left(x^{2}\right)}{30}+R(x),
\end{aligned}
$$

where $x_{i}=i h, h=x / n, f(t)=\exp \left(t^{2}\right)$. 
Let us choose $x=1$. The "exact" value is $E(1)=1.46265174590718$. If we use $(15)$ with $m=10$ then we get $E(1) \approx 1.46265174590708$. If we use (16) with $n=50$ then we get $E(1) \approx 1.46265174590709$. All calculations are done in double precision arithmetic. The first approximate result is obtained faster than the second one. This is a consequence of the fact that we have to calculate the function $\exp \left(t^{2}\right)$ many times when we apply the compound formula and we have only to calculate $\exp \left(x^{2}\right)$ when we apply the summation formula. (In fact, it is possible to find examples where the summation formula is few dozen times faster than the compound formula.)

Similar summation formulas can be obtained for the integrals (special functions): $\int_{0}^{x}[(\sin t) / t] d t, \int_{0}^{x}[(\cos t-1) / t] d t, \int_{0}^{x} \exp \left(-t^{2}\right) d t$, etc.

\section{References}

[1] G. A. Anastassiou, Ostrowski type inequalities, Proc. Amer. Math. Soc., Vol. 123(12), (1995), 3775-3781. E2

[2] P. Cerone and S. S. Dragomir, Midpoint-type rules from an inequalities point of view, handbook of analytic-computational methods in applied mathematics, Editor: G. Anastassiou, CRC Press, New York, (2000), 135-200. E2

[3] P. Cerone and S. S. Dragomir, Trapezoidal-type rules from an inequalities point of view, handbook of analytic-computational methods in applied mathematics, Editor: G. Anastassiou, CRC Press, New York, (2000), 65-134. E2

[4] Lj. Dedić, M. Matić and J. Pečarić, On Euler trapezoid formulae, Appl. Math. Comput., 123 (2001), 37-62.

http://dx.doi.org/10.1016/S0096-3003(00)00054-0 E2 
[5] C. E. M. Pearce, J. Pečarić, N. Ujević and S. Varošanec, Generalizations of some inequalities of Ostrowski-Grüss type, Math. Inequal. Appl., 3(1), (2000), 25-34. http://www.mia-journal.com/files/3-1/full/03-03.PDF E2

[6] N. Ujević and A. J. Roberts, A corrected quadrature formula and applications, ANZIAM J., 45(E), (2004), E41-E56. http://anziamj.austms.org.au/V45/E051 E3 